\title{
A weighted pointwise ergodic theorem
}

by

\section{ASSANI}

Department of Mathematics, University of North Carolina at Chapel Hill, Chapel Hill, NC 27599

ABSTRACT. - We prove the following weighted ergodic theorem:

Let $\left(X_{n}\right)$ be an i.i.d. sequence of symmetric random variables such that $\mathbb{E}\left(\left|X_{1}\right|^{p}\right)<\infty$ for some $p, 1<p<\infty$. Then there exists a set of full measure $\widetilde{\Omega}$ such that for $\omega \in \widetilde{\Omega}$ the following holds:

For all dynamical systems $(Y, \mathcal{G}, \nu, S)$, for all $r, 1<r \leq \infty$ and $g \in L^{r}(\nu)$ the averages $\frac{1}{N} \sum_{n=1}^{N} X_{n}(\omega) g\left(S^{n} y\right)$ converge a.e. $\nu$. $\subseteq$ Elsevier, Paris

RÉSUMÉ. - On obtient le théorème ergodique pondéré suivant : Soit $\left(X_{n}\right)$ une suite de variables aléatoires symmétriques indépendantes identiquement distribuées telles que $\mathbb{E}\left(\left|X_{1}\right|^{p}\right)<\infty$ pour un nombre $p, 1<p<\infty$. Il existe un ensemble de mesure pleine $\widetilde{\Omega}$ tel que si $\omega \in \widetilde{\Omega}$ alors, pour tout système dynamique $(Y, \mathcal{G}, \nu, S)$, pour tout $r, 1<r \leq \infty$ et pour tout $g \in L^{r}(\nu)$ les moyennes $\frac{1}{N} \sum_{n=1}^{N} X_{n}(\omega) g\left(S^{n} y\right)$ convergent p.s. $\nu$. (C) Elsevier, Paris

\section{INTRODUCTION}

Let $\left(X_{n}\right)$ be a stationary process on the probability measure space $(\Omega, \mathcal{F}, \mu)$. The problem of the a.e. convergence with respect to the $y$ variable of the averages $\frac{1}{N} \sum_{n=1}^{N} X_{n}(\omega) g\left(S^{n} y\right)$ has received a significant interest, initiated in part by Bourgain's return times theorem (see [2]

Research supported in part by NSF Grant \#DMS 9305754

1991 Mathematics Subject Classification. Primary 28 D 05, Secondary 60 G 50. 
for one of the proofs of this result). The function $g$ is defined on the dynamical system $(Y, \mathcal{G}, \nu, S)$. The subtlety of these results is that a typical sampling will make these averages a.e. convergent simultaneously for all dynamical systems and all $g$ in some $L^{q}$ spaces.This makes this sampling typical for an uncountable set of dynamical systems and functions. Such a result strengthens greatly the classical Birkhoff theorem applied to the product of two dynamical systems. As far as we know all results of this kind "respected" the duality $\left(L^{p}, L^{q}\right)$; if the $\left(X_{n}\right)$ satisfy the condition $\mathbb{E}\left(\left|X_{1}\right|^{p}\right)<\infty$ for some $p, 1 \leq p \leq \infty$ then the functions $g$ are in $L^{q}$ where $\frac{1}{p}+\frac{1}{q}=1$. The reason for this has been the "apparent" limit imposed by the use of Holder's inequality

$$
\left|\frac{1}{N} \sum_{n=1}^{N} a_{n} b_{n}\right| \leq\left(\frac{1}{N} \sum_{n=1}^{N}\left|a_{n}\right|^{p}\right)^{\frac{1}{p}}\left(\frac{1}{N} \sum_{n=1}^{N}\left|b_{n}\right|^{q}\right)^{\frac{1}{q}} .
$$

In [1] we showed the following result: given $(X, \mathcal{F}, \mu, \varphi)$ and $f \in$ $L^{p}(\mu), 1<p \leq \infty$, there exists a set of full measure $X_{f}$ in $X$ such that for each $x \in X_{f}$ for all probability measure spaces $(Y, \mathcal{G}, \nu)$ and all sequences $\left(Z_{n}\right)$ of independent identically distributed (i.i.d) random variables such that $\mathbb{E}\left(\left|Z_{1}\right|\right)<\infty$, the averages $\frac{1}{N} \sum_{n=1}^{N} f\left(\varphi^{n} x\right) Z_{n}(y)$ converge a.e. $(\nu)$. The sampling $f\left(\varphi^{n} x\right)$ is a.e. typical for the class of all iid random variables with a break of the duality.

In this paper we want to show that this duality can be "broken" for stationary processes where the $\left(X_{n}\right)$ are i.i.d symmetric random variables and the sampling a.e. obtained is typical for the class of all dynamical systems. Moving from the class of all iid random variables to the one of all dynamical systems is not just a technicality. It is known that there are results (such as maximal inequalities) which hold for one class and not the other. In this paper we prove a lemma (lemma 5) that holds for iid random variables but is false for the larger class of all dynamical systems.

Our main theorem is the following result.

THEOREM 1. - Let $\left(X_{n}\right)$ be a sequence of i.i.d. symmetric random variables on the measure space $(\Omega, \mathcal{F}, \mu)$ such that $\mathbb{E}\left(\left|X_{1}\right|^{p}\right)<\infty$ for some $p, 1<p<\infty$. There exists a set of full measure $\widetilde{\Omega} \subset \Omega$ such that for $\omega \in \widetilde{\Omega}$ the following holds:

for all dynamical systems $(Y, \mathcal{G}, \nu, S)$, for all $r, 1<r<\infty$, and for all $g \in L^{r}(\nu)$ the averages

$$
\frac{1}{N} \sum_{n=1}^{N} X_{n}(\omega) \cdot g\left(S^{n} y\right) \text { converges a.e. }(\nu) \text { to } 0 .
$$


The proof of Theorem 1 relies mainly on simple probabilistic methods and the spectral theorem. We do not use Bourgain's theorem or the methods used to prove his theorem. For $\mathrm{p}=2$ and $r \geq 2$ Theorem 1 can be derived from the return times property established for Lebesgue spectrum transformations in [3]. This result was obtained prior to the proof for all dynamical systems obtained by J. Bourgain. At the present time we do not know if Theorem 1 holds for $p=1$ and $r=1$, but the following result (obtained in 1990) seems to indicate that such extension might be true.

THEOREM 2. - Let $(X, \mathcal{F}, \mu, \varphi)$ be an ergodic dynamical system on the finite measure space $(X, \mathcal{F}, \mu)$ and $f \in L^{1}(\mu)$. There exists a set of full measure $\mathcal{X}_{f}$ such that if $x \in \mathcal{X}_{f}$ then we have the following:

the averages $\frac{1}{N} \sum_{n=1}^{N} f\left(\varphi^{n} x\right) g\left(S^{n} y\right)$ converge in $L^{1}(\nu)$ norm for all ergodic dynamical systems $(Y, \mathcal{G}, \nu, S)$ and all $g \in L^{1}(\nu)$.

This result shows that at the norm level the convergence holds for pairs of $\left(L^{1}, L^{1}\right)$ functions.

Proof of Theorem 1. - In the following inequalities the constant $C_{\omega}$ may change from one line to another, but unless specified otherwise it will remain absolute. First we have the following lemma consequence of Salem and Zygmund's maximal inequality [5].

LEMMA 3. - Let $\left(X_{n}\right)$ be a sequence of independent symmetric random variables defined on $(\Omega, \mathcal{F}, \mu)$. We have

$$
\sup _{N} \frac{\sup _{\epsilon}\left|\frac{1}{N} \sum_{n=1}^{N} X_{n}(\omega) e^{i n \epsilon}\right|^{2}}{(\log N) \frac{\left[\sum_{n=1}^{N}\left|X_{n}(\omega)\right|^{2}\right]}{N^{2}}}<\infty \quad \text { Ha.e. }
$$

(ii) For $1<p \leq 2$, for $\mu$ a.e. $\omega$, there exists a finite constant $C_{\omega}$ such that for any $\beta$ and $N$ positive integer we have

$$
\sup _{\epsilon}\left|\frac{1}{N^{\beta}} \sum_{n=1}^{N^{\beta}} X_{n}(\omega) e^{i n \epsilon}\right| \leq C_{\omega}(\log N)^{\frac{1}{2}} \frac{1}{N^{\beta \frac{(p-1)}{p}}} \sup _{M}\left(\frac{1}{M^{\beta}} \sum_{n=1}^{M^{\beta}}\left|X_{n}(\omega)\right|^{p}\right)^{\frac{1}{p}} .
$$

Proof. - We denote by $\left(r_{n}\right)$ the Rademacher functions defined on $(Z, \mathcal{A}, m)$. For $\omega$ fixed, the variables : $z \rightarrow X_{n}(\omega) r_{n}(z)$ are independent and symmetric. By Salem and Zygmund's theorem 4.3.1 [5], we have

$$
m\left\{z: \sup _{N} \frac{\sup _{\epsilon}\left|\frac{1}{N} \sum_{n=1}^{N} X_{n}(\omega) r_{n}(z) e^{i n \epsilon}\right|^{2}}{(\log N)\left(\frac{\sum_{n=1}^{N}\left|X_{n}(\omega)\right|^{2}}{N^{2}}\right)}<\infty\right\}=1 .
$$


This implies that

$$
\mu \otimes m\left\{(\omega, z): \sup _{N} \frac{\sup _{\epsilon}\left|\frac{1}{N} \sum_{n=1}^{N} X_{n}(\omega) r_{n}(z) e^{i n \epsilon}\right|^{2}}{(\log N)\left(\frac{\sum_{n=1}^{N}\left|X_{n}(\omega)\right|^{2}}{N^{2}}\right)}<\infty\right\}=1 .
$$

By Fubini, the last inequality in turn implies that

$$
m \text { a.e. } \mu\left\{\omega: \sup _{N} \frac{\sup _{\epsilon}\left|\frac{1}{N} \sum_{n=1}^{N} X_{n}(\omega) r_{n}(z) e^{i n \epsilon}\right|^{2}}{(\log N)\left(\frac{\sum_{n=1}^{N}\left|X_{n}(\omega)\right|^{2}}{N^{2}}\right)}<\infty\right\}=1 .
$$

As the $X_{n}$ are symmetric and independent we conclude that

$$
\mu\left\{\omega: \sup _{N} \frac{\sup _{\epsilon}\left|\frac{1}{N} \sum_{n=1}^{N} X_{n}(\omega) e^{i n \epsilon}\right|^{2}}{(\log N)\left[\frac{\sum_{n=1}^{N}\left|X_{n}(\omega)\right|^{2}}{N^{2}}\right]}<\infty\right\}=1
$$

Hence for $\mu$ a.e. $\omega$ there exists a finite constant $C_{\omega}$ such that

$$
\sup _{N} \frac{\sup _{\epsilon}\left|\frac{1}{N} \sum_{n=1}^{N} X_{n}(\omega) e^{i n \epsilon}\right|^{2}}{(\log N)\left(\frac{\sum_{n=1}^{N}\left|X_{n}(\omega)\right|^{2}}{N^{2}}\right)}=C_{\omega}<\infty .
$$

This proves (i).

It remains to prove (ii). We have

$$
\begin{aligned}
\left(\frac{\sum_{n=1}^{N}\left|X_{n}(\omega)\right|^{2}}{N^{2}}\right)^{1 / 2} & =\frac{1}{N}\left(\sum_{n=1}^{N}\left|X_{n}(\omega)\right|^{2}\right)^{\frac{1}{2}} \leq \frac{1}{N}\left(\sum_{n=1}^{N}\left|X_{n}(\omega)\right|^{p}\right)^{\frac{1}{p}} \\
& =\frac{1}{N^{\frac{p-1}{p}}}\left(\frac{\sum_{n=1}^{N}\left|X_{n}(\omega)\right|^{p}}{N}\right)^{\frac{1}{p}}
\end{aligned}
$$

So using $(*)$ we conclude that for any $\beta$ and $N$ positive integers we have

$$
\begin{aligned}
& \sup _{\epsilon}\left|\frac{1}{N^{\beta}} \sum_{n=1}^{N^{\beta}} X_{n}(\omega) e^{i n \epsilon}\right| \\
& \quad \leq C_{\omega}(\log N)^{\frac{1}{2}}\left(\sup _{M}\left(\frac{\sum_{n=1}^{M^{\beta}}\left|X_{n}(\omega)\right|^{p}}{M^{\beta}}\right)^{\frac{1}{p}} \cdot \frac{1}{N^{\beta} \frac{(p-1)}{p}}\right) .
\end{aligned}
$$

This concludes the proof of Lemma 3. 
We denote by $\widetilde{\Omega}$ the intersection of the sets where $C_{\omega}<\infty$ and $\sup _{N}\left(\frac{\sum_{n=1}^{N}\left|X_{n}(\omega)\right|^{p}}{N}\right)<\infty$. Under the hypothesis of Theorem 1 , the set $\widetilde{\Omega}$ has full measure.

Lemma 4. - For $\omega \in \widetilde{\Omega}$ there exists $\beta$ positive integer such that

$$
\int \sum_{N=1}^{\infty}\left|\frac{1}{N^{\beta}} \sum_{n=1}^{N^{\beta}} X_{n}(\omega) g\left(S^{n} y\right)\right| d \nu<\infty
$$

for all dynamical systems $(Y, \mathcal{G}, \nu, S)$ and for all $g \in L^{r}(\nu), 1<r<\infty$.

Proof. - Once again, it is enough to consider the case $1<r<2$ and $g \geq 0$. The general case follows by considering the functions $g^{+}$and $g^{-}$.

Given $g \in L^{r}(\nu)$ we denote by $g_{N}^{1}$ the function $g \wedge K_{N}$ and by $g_{N}^{2}$ the function $g-g_{N}^{1}$. The constant $K_{N}$ will be specified later. We have

$$
\begin{aligned}
& \sum_{N=1}^{\infty} \int\left|\frac{1}{N^{\beta}} \sum_{n=1}^{N^{\beta}} X_{n}(w) g_{N}^{1}\left(S^{n} y\right)\right| d \nu \\
& \leq \sum_{N=1}^{\infty}\left(\int\left|\frac{1}{N^{\beta}} \sum_{n=1}^{N^{\beta}} X_{n}(\omega) g_{N}^{1}\left(S^{n} y\right)\right|^{2} d \nu\right)^{\frac{1}{2}} \\
& =\sum_{N=1}^{\infty}\left(\int\left|\frac{1}{N^{\beta}} \sum_{n=1}^{N^{\beta}} X_{n}(\omega) e^{i n \epsilon}\right|^{2} d \sigma_{g_{N}^{1}}\right)^{\frac{1}{2}}
\end{aligned}
$$

(where $\sigma_{g_{N}^{1}}$ is the spectral measure of $g_{N}^{1}$ with respect to the dynamical system $(Y, \mathcal{G}, \nu, S))$

$$
\begin{aligned}
& \leq \sum_{N=1}^{\infty}\left(\sup _{\epsilon}\left|\frac{1}{N^{\beta}} \sum_{n=1}^{N^{\beta}} X_{n}(\omega) e^{i n \epsilon}\right|^{2}\left\|g_{N}^{1}\right\|_{2}^{2}\right)^{\frac{1}{2}} \\
& =\sum_{N=1}^{\infty} \sup _{\epsilon}\left|\frac{1}{N^{\beta}} \sum_{n=1}^{N^{\beta}} X_{n}(\omega) e^{i n \epsilon}\right|\left(\int\left|g_{N}^{1}\right|^{2} d \nu\right)^{\frac{1}{2}} \\
& =\sum_{N=1}^{\infty} \sup _{\epsilon}\left|\frac{1}{N^{\beta}} \sum_{n=1}^{N^{\beta}} X_{n}(\omega) e^{i n \epsilon}\right|\left(\int\left|g_{N}^{1}\right|^{r}\left|g_{N}^{1}\right|^{2-r} d \nu\right)^{\frac{1}{2}} \\
& \leq \sum_{N=1}^{\infty} \sup _{\epsilon}\left|\frac{1}{N^{\beta}} \sum_{n=1}^{N^{\beta}} X_{n}(\omega) e^{i n \epsilon}\right| K_{N}^{\frac{2-r}{2}}\left(\int\left|g_{N}^{1}\right|^{r} d \nu\right)^{\frac{1}{2}} \\
& \leq \sum_{N=1}^{\infty} \sup _{\epsilon}\left|\frac{1}{N^{\beta}} \sum_{n=1}^{N^{\beta}} X_{n}(\omega) e^{i n \epsilon}\right| K_{N}^{\frac{2-r}{2}}\|g\|_{r}^{\frac{r}{2}}
\end{aligned}
$$


By Lemma 3 this last sum is less than

(A)

$$
\begin{aligned}
& C_{\omega} \cdot\left(\sum_{N=1}^{\infty}\left(\frac{1}{N^{\beta\left(\frac{p-1}{p}\right)}} K_{N}^{\frac{2-r}{2}}\right)\right) \\
& \cdot(\log N)^{\frac{1}{2}} \sup _{M}\left(\frac{\sum_{n=1}^{M^{\beta}}\left|X_{n}(\omega)\right|^{p}}{M^{\beta}}\right)^{1 / p}\|g\|_{r}^{1 / 2} .
\end{aligned}
$$

It remains to control the second sum

$$
\sum_{N=1}^{\infty} \int\left|\frac{1}{N^{\beta}} \sum_{n=1}^{N^{\beta}} X_{n}(\omega) g_{N}^{2}\left(S^{n} y\right)\right| d \nu(y)
$$

This sum is less than

$$
\begin{aligned}
& \sum_{N=1}^{\infty}\left(\frac{1}{N^{\beta}} \sum_{n=1}^{N^{\beta}}\left|X_{n}(\omega)\right| \cdot \int\left|g_{N}^{2}\right|\left(S^{n} y\right) d \nu\right) \\
& \quad \leq \sum_{N=1}^{\infty} \sup _{M}\left(\frac{1}{M^{\beta}} \sum_{n=1}^{M^{\beta}}\left|X_{n}(\omega)\right|\right)\left\|g_{N}^{2}\right\|_{1} \\
& \quad \leq \sup _{M}\left(\frac{1}{M^{\beta}} \sum_{n=1}^{M^{\beta}}\left|X_{n}(\omega)\right|\right) \sum_{N=1}^{\infty} \sum_{i=K_{N}}^{\infty} i \nu\left(A_{i}\right)
\end{aligned}
$$

where $A_{i}=\{y: i-1<g \leq i\}$. We have

$$
\begin{aligned}
\sum_{N=1}^{\infty} \sum_{i=K_{N}}^{\infty} i \nu\left(A_{i}\right) & \leq \sum_{N=1}^{\infty} N \sum_{i=K_{N}}^{K_{N+1}} i \nu\left(A_{i}\right) \\
& \leq \sum_{N=1}^{\infty} N K_{N+1} \sum_{i=K_{N}}^{K_{N+1}} \nu\left(A_{i}\right) \\
& \leq \sum_{N=1}^{\infty}(N+1) K_{N+1} \nu\left\{K_{N}<g \leq K_{N+1}\right\}
\end{aligned}
$$


It is time to specify $K_{N}$. We choose $K_{N}$ an increasing sequence of integers such that $K_{N} \sim N^{1 / r-1}$. This implies that

$$
\begin{aligned}
& \sum_{N=1}^{\infty}(N+1) K_{N+1} \nu\left\{K_{N}<g \leq K_{N+1}\right\} \\
& \quad \sim \sum_{N=1}^{\infty}(N+1)^{r / r-1} \nu\left\{N^{1 / r-1}<g \leq(N+1)^{1 / r-1}\right\} \\
& \quad=\sum_{N=1}^{\infty}(N+1)^{r / r-1} \nu\left\{N^{r / r-1}<g^{r} \leq(N+1)^{r / r-1}\right\} \\
& \quad \sim \int g^{r} d \nu .
\end{aligned}
$$

Finally, it remains to show that such a choice of $K_{N}$ is compatible with (A).

But this is now easy. We can always choose $\beta$ large enough so that

$$
\frac{N^{\left(\frac{2-r}{2}\right)\left(\frac{1}{r-1}\right)}}{N^{\beta\left(\frac{p-1}{p}\right)}} \leq \frac{1}{N^{\gamma}} \quad \text { for } \gamma \text { integer } \gamma \geq 2 .
$$

(In fact we will select $\beta \geq 3$ such that $\frac{\beta-2}{\beta} \geq \frac{1}{p}$.) This ends the proof of Lemma 4.

By Lemma 4 we have found a set $\widetilde{\Omega}$ of full measure, $\beta \geq 3$, such that for $\omega \in \widetilde{\Omega}$ we have

$$
\frac{1}{N^{\beta}} \sum_{n=1}^{N^{\beta}} X_{n}(\omega) g\left(S^{n} y\right) \underset{N}{\rightarrow} 0 \quad \text { a.e. }(\nu)
$$

for all dynamical systems $(Y, \mathcal{G}, \nu, S))$ and all $g \in L^{r}(\nu), 1<r<\infty$. We need, to conclude the proof of Theorem 1, to prove the same result along the sequence of all integers.

To achieve this result we will further restrict the set $\widetilde{\Omega}$, by considering the following set

$$
\Omega^{\prime}=\left\{\omega \in \widetilde{\Omega}: \sup _{N} \frac{\sum_{n=N^{\beta}}^{(N+1)^{\beta}}\left|X_{n}(\omega)\right|}{N^{\beta-1}}<\infty\right\} .
$$

In our next lemma we are going to prove that this set $\Omega^{\prime}$ also has full measure. This lemma does not hold for general stationary processes [see [3], for instance].

Vol. $34, n^{\circ}$ 1-1998. 
LEMMA 5. - Let $\beta$ be a positive integer, $\beta \geq 3$, and $\left(Y_{n}\right)$ a sequence of i.i.d. random variables such that $\mathbb{E}\left(\left|Y_{1}\right|^{p}\right)<\infty$. The sequence

$$
\frac{1}{N^{\beta-1}} \sum_{n=N^{\beta}}^{(N+1)^{\beta}} Y_{n}(w)
$$

converges a.e. to $C(\beta) \cdot \mathbb{E}\left(Y_{1}\right)$ where $C(\beta)$ is an absolute constant depending only on $\beta$, for $\frac{\beta-2}{\beta} \geq \frac{1}{p}$.

Proof of Lemma 5. - We again use the versatile method of truncation by introducing the variables $Z_{n}$ and $T_{n}$;

$$
Z_{n}(\omega)=Y_{n}(\omega) \wedge n^{((\beta-2) / \beta)}, T_{n}(\omega)=Y_{n}(\omega)-Z_{n}(\omega)
$$

For each $\epsilon>0$ we have by Chebychev's inequality

$$
\begin{aligned}
& \sum_{N=1}^{\infty} \mu\left\{\omega:\left|\frac{\sum_{n=N^{\beta}}^{(N+1)^{\beta}}\left(Z_{n}(\omega)-\mathbb{E}\left(Z_{n}\right)\right)}{N^{\beta-1}}\right|>\epsilon\right\} \\
& \quad \leq \frac{1}{\epsilon^{2}} \sum_{N=1}^{\infty} \frac{\sum_{n=N^{\beta}}^{(N+1)^{\beta}} \operatorname{var}\left(Z_{n}-\mathbb{E}\left(Z_{n}\right)\right)}{N^{2(\beta-1)}} \\
& \quad \leq \frac{1}{\epsilon^{2}} \sum_{N=1}^{\infty} \frac{(N+1)^{\beta}-N^{\beta}}{N^{2(\beta-1)}} \int Z_{(N+1)^{\beta}}^{2}(\omega) d \mu
\end{aligned}
$$

$\left(\right.$ as $\operatorname{var}\left(Z_{n}-\mathbb{E}\left(Z_{n}\right) \leq \int Z_{n}^{2} d \mu \leq \int Z_{(N+1)^{\beta}}^{2} d \mu\right.$ for $\left.N^{\beta} \leq n \leq(N+1)^{\beta}\right)$,

$$
\begin{aligned}
& =\frac{1}{\epsilon^{2}} \sum_{N=1}^{\infty} \frac{(N+1)^{\beta}-N^{\beta}}{N^{2(\beta-1)}} \int Y_{1}^{2}(\omega) \mathbf{1}_{\left\{Y_{1}<(N+1)^{\beta-2}\right\}} d \mu \\
& \leq \frac{C(\beta)}{\epsilon^{2}} \sum_{N=1}^{\infty} \frac{1}{N^{\beta-1}} \sum_{j=1}^{N+1} \int Y_{1}^{2}(\omega) \mathbf{1}_{\left\{(j-1)^{\beta-2} \leq Y_{1}<j^{\beta-2}\right\}} d \mu \\
& \leq \frac{C(\beta)}{\epsilon^{2}} \sum_{N=1}^{\infty} \int Y_{1}^{2}(\omega) \mathbf{1}_{\left\{(N-1)^{\beta-2} \leq Y_{1}<N^{\beta-2}\right\}} \cdot\left(\sum_{j=N}^{\infty} \frac{1}{j^{\beta-1}}\right) d \mu \\
& \leq \frac{C^{\prime}(\beta)}{\epsilon^{2}} \sum_{N=1}^{\infty} \int Y_{1}^{2} \frac{\mathbf{1}_{\left\{(N-1)^{\beta-2} \leq Y_{1}<N^{\beta-2}\right\}}}{N^{\beta-2}} d \mu \\
& \leq \frac{C^{\prime}(\beta)}{\epsilon^{2}} \sum_{N=1}^{\infty} \int Y_{1}(w) \mathbf{1}_{\left\{(N-1)^{\beta-2} \leq Y_{1}<N^{\beta-2}\right\}} d \mu \\
& \leq \frac{C^{\prime}(\beta)}{\epsilon^{2}} \int Y_{1}(\omega) d \mu=\frac{C^{\prime}(\beta)}{\epsilon^{2}} \mathbb{E}\left(Y_{1}\right) .
\end{aligned}
$$


This implies that the sequence

$$
\frac{\sum_{n=N^{\beta}}^{(N+1)^{\beta}}\left(Z_{n}(\omega)-\mathbb{E}\left(Z_{n}\right)\right)}{N^{\beta-1}}
$$

converges to 0 a.e. As $\mathbb{E}\left(Z_{n}\right) \rightarrow \mathbb{E}\left(Y_{1}\right)$ we also have the a.e. convergence of the sequence

$$
\frac{\sum_{n=N^{\beta}}^{(N+1)^{\beta}} Z_{n}(\omega)}{N^{\beta-1}}
$$

Finally it remains to evaluate the averages

$$
\frac{1}{N^{\beta-1}} \sum_{n=N^{\beta}}^{(N+1)^{\beta}} T_{n}(\omega)
$$

We have $\mu\left\{T_{n} \neq 0\right\}=\mu\left\{Y_{1}>n^{\frac{\beta-2}{\beta}}\right\} \leq \mu\left\{Y_{1}>n^{\frac{1}{p}}\right\}$. This last term is the generic term of a convergent series as $Y_{1} \in L^{p}$. Thus $\mu$ a.e. $T_{n}$ is equal to zero for $n$ large enough.

\section{END OF THE PROOF OF THEOREM 1}

We know that $\frac{1}{N^{\beta}} \sum_{n=1}^{N^{\beta}} X_{n}(\omega) g\left(S^{n} y\right)$ converges to 0 a.e. for all dynamical systems $(Y, \mathcal{G}, \nu, S)$ and all $g \in L^{r}(\nu), 1<r<\infty$.

We also know that

$$
\sup _{N} \frac{1}{N^{\beta-1}} \sum_{n=N^{\beta}}^{(N+1)^{\beta}}\left|X_{n}(\omega)\right| \leq C_{\omega}<\infty
$$

for $\omega \in \Omega^{\prime}$ with $\mu\left(\Omega^{\prime}\right)=1$ (by Lemma 4 and 5). In particular, we have for all $N$

$$
\frac{\sum_{n=N^{\beta}}^{(N+1)^{\beta}}\left|X_{n}(\omega)\right|}{N^{\beta}} \leq \frac{C_{\omega}}{N} .
$$

For each positive integer $M$ we can find an integer $N$ such that

$$
N^{\beta} \leq M<(N+1)^{\beta}
$$

Vol. $34, \mathrm{n}^{\circ} 1-1998$. 
We have

$$
\begin{aligned}
& \left|\frac{1}{M} \sum_{n=1}^{M} X_{n}(\omega) g\left(S^{n} y\right)\right| \\
& \quad \leq\left|\frac{N^{\beta}}{N^{\beta} M} \sum_{n=1}^{N^{\beta}} X_{n}(\omega) g\left(S^{n} y\right)\right|+\frac{1}{M} \sum_{n=N^{\beta}}^{(N+1)^{\beta}}\left|X_{n}(\omega)\right|\left|g\left(S^{n} y\right)\right| .
\end{aligned}
$$

The first term converges a.e. to 0 by Lemma 4 . The second term is dominated by

$$
\frac{\sum_{n=N^{\beta}}^{(N+1)^{\beta}}\left|X_{n}(\omega)\right|\left|g\left(S^{n} y\right)\right|}{N^{\beta}}
$$

We want to show that this last term tends also to 0 a.e. $(\nu)$ for $\omega \in \Omega^{\prime}$.

We consider $g \geq 0$,

$$
g_{N}=g \wedge N^{1 / r} \text { and } h_{N}=g-g_{N}
$$

We have

$$
\begin{aligned}
& \frac{\sum_{n=N^{\beta}}^{(N+1)^{\beta}}\left|X_{n}(\omega)\right|\left|g\left(S^{n} y\right)\right|}{N^{\beta}} \\
& \quad \leq \frac{\sum_{n=N^{\beta}}^{(N+1)^{\beta}}\left|X_{n}(\omega)\right|\left[g_{N}\left(S^{n} y\right)\right]}{N^{\beta}}+\frac{\sum_{n=N^{\beta}}^{(N+1)^{\beta}}\left|X_{n}(\omega)\right| h_{N}\left(S^{n} y\right)}{N^{\beta}} .
\end{aligned}
$$

The first term is less than

$$
\frac{\sum_{n=N^{\beta}}^{(N+1)^{\beta}}\left|X_{n}(\omega)\right| N^{1 / r}}{N^{\beta}} \leq \frac{C_{\omega} N^{1 / r}}{N} \underset{N}{\rightarrow} 0
$$

We integrate the second term with respect to $(\nu)$ to get

$$
\begin{aligned}
\frac{\sum_{n=N^{\beta}}^{(N+1)^{\beta}}\left|X_{n}(\omega)\right| \int h_{N}(y) d \nu}{N^{\beta}} & =\sum_{n=N^{\beta}}^{(N+1)^{\beta}} \frac{\left|X_{n}(\omega)\right|}{N^{\beta}} \int g \cdot \mathbf{1}_{\left\{g>N^{1 / r}\right\}} d \nu \\
& <C_{\omega} \int g \frac{\mathbf{1}_{\left\{g>N^{1 / r}\right\}}}{N} d \nu
\end{aligned}
$$


Hence

$$
\begin{aligned}
& \sum_{N=1}^{\infty} \frac{\sum_{n=N^{\beta}}^{(N+1)^{\beta}}\left|X_{n}(\omega)\right| \int h_{N} d \nu}{N^{\beta}} \\
& \leq C_{\omega} \sum_{N=1}^{\infty} \int g \cdot \frac{\mathbf{1}_{\left\{g>N^{1 / r}\right\}}}{N} d \nu \\
& \leq C_{\omega} \int \sum_{N=1}^{\infty} \frac{g}{N} \mathbf{1}_{\left\{g>N^{1 / r}\right\}} d \nu \\
& \leq C_{\omega} \int\left(\sum_{N=1}^{\infty} \frac{g^{r}}{N^{r}}\right)^{1 / r}\left(\sum_{N=1}^{\infty} \mathbf{1}_{\left\{g>N^{1 / r}\right\}}\right)^{1 / r^{*}} d \nu \quad\left(\text { where } \frac{1}{r}+\frac{1}{r^{*}}=1\right) \\
& \leq C_{\omega}\left(\int\left(\sum_{N=1}^{\infty} \frac{g^{r}}{N^{r}}\right) d \nu\right)^{1 / r}\left(\int \sum_{N=1}^{\infty} 1_{\left\{g>N^{1 / r}\right\}} d \nu\right)^{1 / r^{*}} \\
& =C_{\omega}\left(\int g^{r} d \nu\right)^{1 / r}\left(\sum_{N=1}^{\infty} \nu\left\{g^{r}>N\right\}\right)^{1 / r^{*}} \\
& <\infty \text { as } g \in L^{r}(\nu) \text {. }
\end{aligned}
$$

This implies the a.e. convergence to 0 of

$$
\frac{\sum_{n=N^{\beta}}^{(N+1)^{\beta}}\left|X_{n}(\omega)\right|\left|h_{N}\right|\left(S^{n} y\right)}{N^{\beta}}
$$

and ends the proof of Theorem 1.

Proof of Theorem 2. - By the Wiener-Wintner ergodic theorem we know that given $f \in L^{1}(\mu)$ there exists a set $X_{f}$ of full measure such that for $x \in$ $X_{f}$ the averages $\frac{1}{N} \sum_{n=1}^{N} f\left(\varphi^{n} x\right) e^{i n \epsilon}$ converge for all $\epsilon$. An application of the spectral theorem then implies that for $x \in X_{f}$ for all ergodic dynamical systems $(Y, \mathcal{G}, \nu, S)$ for all $g \in L^{2}(\nu)$ the averages $\frac{1}{N} \sum_{n=1}^{N} f\left(\varphi^{n} x\right) g \circ S^{n}$ converge in $L^{2}(\nu)$ norm. We will obtain the convergence for all $g \in L^{1}(\nu)$ if the operators $T_{N}^{x}: g \in L^{1}(\nu) \rightarrow \frac{1}{N} \sum_{n=1}^{N} f\left(\varphi^{n} x\right) g \circ S^{n}$ are uniformly bounded in $L^{1}$. This follows from Birkhoff's pointwise ergodic theorem: the norm of $T_{N}^{x}$ is

$$
\left\|T_{N}^{x}\right\|_{1} \leq \frac{1}{N} \sum_{n=1}^{N}\left|f\left(\varphi^{n} x\right)\right|
$$

Thus

$$
\sup _{N}\left\|T_{N}^{x}\right\|_{1} \leq \sup _{N} \frac{1}{N} \sum_{n=1}^{N}\left|f\left(\varphi^{n} x\right)\right| .
$$

Vol. $34, \mathrm{n}^{\circ} 1-1998$. 
By taking $x$ in the set $X_{f} \cap \tilde{X}_{f}$ where on $\tilde{X}_{f}$ we have $\sup _{N} \frac{1}{N} \sum_{n=1}^{N}\left|f\left(\varphi^{n} x\right)\right|<\infty$ we obtain a set of full measure for which Theorem 2 holds.

Remark. - The interested reader can verify that the arguments we used apply equally well to the following averages $\frac{1}{N} \sum_{n=1}^{N} X_{n}(\omega) \cdot g\left(S^{p(n)} y\right)$ where $p(n)=n^{2}$ or more generally any positive integer valued polynomial with degree greater or equal to one.

\section{REFERENCES}

[1] I. AssanI, Strong laws for weighted sums of independently identically distributed random variables, Duke Mathematical Journal, Vol. 88, 1997, pp. 217-246.

[2] J. Bourgain, H. Furstenberg, Y. Katznelson and D. Ornstein, Return times of dynamical systems Appendix to J. Bourgain, Pointwise Ergodic Theorems for Arithmetic Sets, I.H.E.S., Vol. 69, 1989, pp. 5-45.

[3] A. Bellow, R. Jones and J. Rosenblatt, Convergence for moving averages, Ergod. Th. \& Dynam. Sys., Vol. 10, 1990, pp. 43-62.

[4] A. Bellow and V. Losert, The weighted pointwise ergodic theorem and the individual ergodic theorem along subsequences, Trans. Amer. Math. Soc., Vol. 288, 1985, pp. 307-345.

[5] R. SALEM and A. ZyGmund, Some properties of trigonometric series whose terms have random signs, Acta. Math., Vol. 91, 1954, pp. 245-301.

(Manuscript received November 13, 1996; Revised September 1, 1997.) 